\title{
Outage performance of low-latency decode-and-forward cooperative wireless networks
}

\author{
Nemanja Zdravkovic ${ }^{1,2^{*}}$, Aleksandra Cvetkovic ${ }^{2}$, Kimmo Kansanen $^{1}$ and Goran T. Djordjevic ${ }^{2}$
}

\begin{abstract}
In this paper, we study a noise-limited low-latency decode-and-forward cooperative network set in a dynamic environment prone to link blockage. We analyse outage probability (OP) performance considering a temporally correlated fading channel between cooperating nodes employing a threshold-based protocol for internode communication. Exact and asymptotic analytical expressions for OP over Rayleigh fading, when maximal-ratio combining is applied at the destination, are derived. Asymptotic results give a better insight into outage performance at high average signal-to-noise ratio (SNR). We analyse the OP dependence on the SNR and link blockage probability and correlation coefficient of the estimated and actual internode SNR. The results illustrate that the internode protocol improves outage performance in the whole range of average uplink SNR. Internode channel correlation has a significant effect on the OP in the range of low average internode SNR. With increasing correlation, the OP increases up to an order of magnitude. The results also show that link blockage has a dominating effect on OP performance. The finite probability of all internode links being blocked reduces network performance to a single-link scenario, regardless of using the threshold-based protocol.
\end{abstract}

Keywords: Cooperative networks, Decode-and-forward relay, Link blockage, Rayleigh fading, Outage probability

\section{Introduction}

In order to improve overall wireless network performance, full spatial diversity can be achieved by node cooperation [1]. Usually, cooperation strategies include amplify-andforward (AF) and decode-and-forward (DF) protocols. This paper considers uncoordinated DF cooperation, due to the fact that AF protocols require channel state information (CSI) between nodes, which cannot be always the case in real-world situations. This kind of cooperation assumes that nodes, which decode data from all or some of their partners, provide assistance in transmission, even if the cooperation is not bidirectional. On the other hand, coordinated DF cooperation guarantees that a node is assisted only by the nodes which that node is assisting. The number of independent data transmission

\footnotetext{
*Correspondence: nemanja.zdravkovic@iet.ntnu.no

1 Department of Electronics and Telecommunications, Norwegian University of Science and Technology - NTNU, Høgskoleringen 1, 7491 Trondheim, Norway

2 Faculty of Electronic Engineering, University of Niš, Aleksandra Medvedeva

14,18000 Niš, Serbia
}

paths in the coordinated protocol is lower than that in the uncoordinated case [1].

Performance analysis of DF networks was presented in [2-13]. Packet error probability approximation when turbo codes are applied was performed in [2], where the expressions for error probability were derived for AF, DF and hybrid AF/DF protocols, using strong forward error correction codes over Rayleigh fading. In [3], clusterbased cooperation was introduced where each cluster acts as a virtual node with multiple antennas. A closed-form expression for outage probability (OP) of DF networks was derived in [4]. Exact expressions for OP and outage capacity in three proactive cooperative diversity schemes were derived in [5], which are valid for an arbitrary network topology and average signal-to-noise ratio (SNR). A limited feedback scheme which improves outage performance for a wireless DF network was presented in [6]. The authors considered both limited-and full-rate feedback assuming imperfect channel estimation at the receiver and derive upper bounds on the OP. Exact analytical expressions for OP of various cooperative DF networks over 
Rayleigh fading for low and high average SNR ranges were presented in [7]. Outage probability performance for both AF and DF cooperative networks was evaluated in [8]. The authors included a new outage analysis for an AF protocol and have concluded that the performance of their proposed DF protocol will be better in the low average SNR regime, while their AF protocol achieves DF performance in high average SNR. Furthermore, increasing the network size will improve outage performance. Performance analysis of adaptive decode-and-forward relaying in non-coherent cooperative networks was analysed in [9]. In [10], the authors considered the performance of distributed space-time coded cooperative relay networks based on inter-relay communications.

Chatzigeorgiou et al. [11] analysed the OP of uncoordinated DF networks where nodes act as both relays and transmitters for the case of Rayleigh fading channels. Transmission is performed cooperatively in two separate stages. In the first stage, a node will broadcast its packet to other nodes and to the destination. In the second stage, relaying nodes will either forward data or copy their own data multiple times to the destination, which applies maximal-ratio combining (MRC). Outage performance analysis over Nakagami- $m$ fading channels, which is a more general fading model, was presented in [12]. In [13], a threshold-based protocol was proposed to improve system performance over Rayleigh fading. The protocol is based on monitoring the channel between nodes and sending data in different time periods depending on the estimated value of the instantaneous SNR.

Our main motivation in this paper is the evaluation of outage performance under conditions found typically in industrial wireless networks, located in large, manmade installations. Apart from the fading phenomena, one should account for macroscopic changes in the propagation environment, like large objects moving to block line-of-sight paths, and other obstacles [14-16]. Within this type of environment network, nodes are subject to dynamic shadowing and outage events occur due to propagation phenomena, which are not only static but are also slower than short-term fading. In this paper, we therefore derive outage performance of the network which includes the probability of internode link blockage. Additionally, for internode communication, we adopt the thresholdbased internode protocol from [13], which improves the overall system performance. However, whereas in [13], the authors assumed independent SNR instances in the initial and delayed transmission in the internode protocol, and we include the analysis of temporal variations of the wireless channel in internode communication. The fading estimate will be different from the actual fading experienced by the packet due to temporal variation of the fading channel, which affects the packet, introducing performance loss. Transmission is performed cooperatively in two separate stages. In the first stage, a node will broadcast its packet to other nodes and to the destination. In the second stage, relaying nodes will either forward the data or copy their own data multiple times to the destination, which applies MRC. The analytical expression for OP in the network with threshold-based protocol over correlated internode fading channel is derived as well.

To the best of the authors' knowledge, the derived closed-form expressions for outage probability are novel and can be utilized in determining outage performance in a dynamic network environment prone to link blockage. The effects of parameters such as the average uplink and internode SNR, the number of nodes in the network and the correlation coefficient on the outage performance are analysed. Moreover, derived asymptotic expressions directly demonstrate the diversity and coding gains of the observed network for different ratios of average internode and uplink SNRs.

The rest of the paper is organized as follows. Section 2 introduces the system model, and the internode link blockage and the threshold-based protocol are presented. In Section 3, the derivation of the probability of successful internode decoding is presented. The outage performance of the network with the internode link blockage and threshold-based protocol is derived in Section 4. Asymptotic behaviour for both average internode and average uplink SNRs are discussed in Section 5. The numerical results with appropriate discussions are given in Section 6, and the concluding remarks are presented in Section 7.

\section{System model}

We investigate a cooperative wireless network which is comprised of $M$ nodes. The nodes, denoted by $U_{i}, i=$ $1, \ldots, M$, are placed around the destination, denoted by $D$, as shown in Fig. 1. The destination can detect each node individually due to orthogonal channels. Channel orthogonality can be achieved by using various modes of multiple access, i.e. time/frequency/code division multiple access (TDMA/FDMA/CDMA). The nodes cooperate and communicate with each other and the destination over a frequency-flat channel subject to Rayleigh fading and additive white Gaussian noise (AWGN). The channel between a node and the destination is denoted as the uplink channel, while the channels between cooperating nodes are denoted as internode channels.

Without loss of generality, we focus on the internode links from a single node, for example $U_{i}$. Due to various obstacles like mobile obstructions, an internode link from $U_{i}$ to $U_{j}$, denoted by $(i \leftrightarrow j)$, can be blocked with probability $b_{i j}, j=1, \ldots, M, j \neq i$. When $(i \leftrightarrow j)$ is blocked, node $U_{j}$ is cut off from the network from the point of view of $U_{i}$, and $U_{i}$ continues to cooperate with the remaining nodes. Internode links are reciprocal; therefore, if $(i \leftrightarrow j)$ is blocked, $(j \leftrightarrow i)$ is blocked as well. We assume that the 


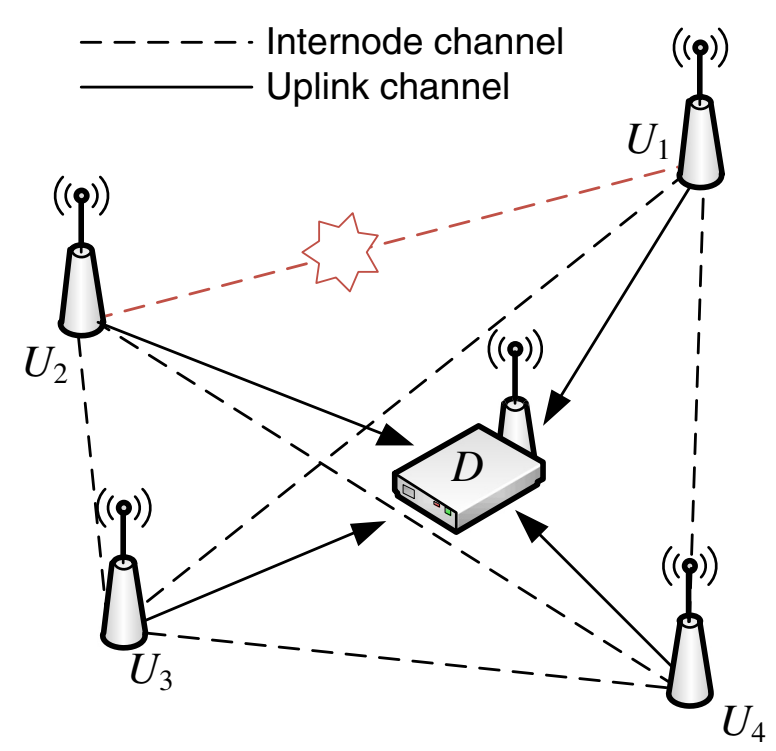

Fig. 1 System model. A diagram depicting a system with four nodes and the destination

environment from nodes to the destination is such that direct links, from $U_{i}$ to $D$, cannot be blocked, and communication from a node to the destination is always possible and all average uplink SNRs are equal.

Cooperation consists of two stages. In the first stage, each node sends its own packet to the destination and to the other nodes. In the second stage, each node acts as a relay, re-encodes and forwards the received packet.

Figure 2a shows the transmissions from node $U_{i}$ to other nodes and to the destination using TDMA. A total of $M$ time slots are used in the first phase of transmission. Note that each packet transmission time is shorter than the dedicated time slot, allowing the use of the internode protocol described in the following paragraph.

For internode communication, we adopt a more precise version of the protocol described in [13]. Each node monitors and estimates the instantaneous internode SNR to a non-blocked link. Depending on the internode CSI, each node sends its packet to other cooperating nodes if the internode SNR is above a predetermined threshold, as shown in Fig. 2b. If this is not the case, regardless of the CSI, each node sends its packet after a waiting period, as in Fig. 2c. In our model, the instantaneous SNR of the delayed transmission will be correlated with the initial estimated SNR value. The random variables associated with the instantaneous internode SNR in the initial and delayed transmission in the internode protocol are denoted as $\gamma_{i j}$ and $\gamma_{i j}{ }^{\prime \prime}$, respectively. It is assumed that $\gamma_{i j}{ }^{\prime}$ and $\gamma_{i j} \prime$ ' are correlated and have a joint probability density function (PDF) given by [17]

$$
p_{\gamma_{i j^{\prime}}, \gamma_{i j^{\prime \prime}}}\left(\gamma_{i j}{ }^{\prime}, \gamma_{i j}{ }^{\prime \prime}\right)=\frac{e^{-\frac{\gamma_{i j^{\prime}}+\gamma_{i j}{ }^{\prime \prime}}{(1-\rho) \bar{\gamma}_{i j}}}}{(1-\rho) \bar{\gamma}_{i j}^{2}} I_{0}\left(\frac{2 \sqrt{\rho \gamma_{i j}{ }^{\prime} \gamma_{i j}{ }^{\prime \prime}}}{(1-\rho) \bar{\gamma}_{i j}}\right) \text {, }
$$

where $\rho$ is the correlation coefficient, assumed to be the same for all links, $\bar{\gamma}_{i j}$ is the average internode SNR for the link $(i \leftrightarrow j)$ and $I_{0}(\cdot)$ is the zeroth order modified Bessel function of the first kind.

At the end of the first stage, each node has received $M-1$ coded packets from other nodes. Out of those received coded packets, $n$ packets are not successfully decoded. Hence, a node which fails to decode $n$ packets has decoded $M-n-1$ packets successfully. During the second stage of cooperation, each node acts as a relay and re-encodes and forwards the $M-n-1$ packets to the destination. To maintain constant cooperation frame energy, the $n$ packets which the node failed to decode are replaced with $n$ copies of its own packet.

At the end of the second stage of cooperation, node $U_{i}$ has transmitted $n+1$ copies of its own packets over the uplink channel. In addition to these packets, $M-n-1$ copies of the same packet are transmitted to the destination over other cooperating nodes. The destination then applies MRC of the $M$ copies of the packet.

If we observe the OP from node $U_{i}$, the number of cooperating nodes will depend on the network size, i.e. the number of unblocked links to $U_{i}$. We assume that link blockages are statistically independent. $n_{b}$ denotes the number of blocked links to node $U_{i}$. The probability of $N_{b}$ links being blocked is given by

$$
\operatorname{Pr}\left(n_{b}=N_{b}\right)=\operatorname{Pr}\left(N_{b}\right)=\sum_{\substack{S_{b} \subseteq S_{i} \\\left|S_{b}\right|=N_{b}}} \prod_{\nu \in S_{i}} b_{i v} \prod_{\mu \in S_{i} \backslash S_{b}}\left(1-b_{i \mu}\right),
$$

with $N_{b}=1,2, \ldots, M-1, S_{i}$ denoting the set of internode links from $U_{i}, S_{b}$ the subset of blocked links, $\left|S_{b}\right|$ the 


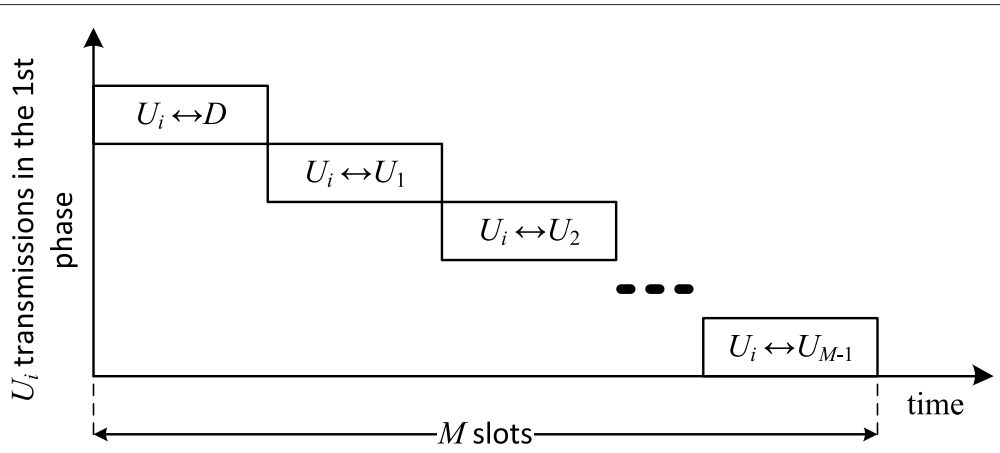

(a)

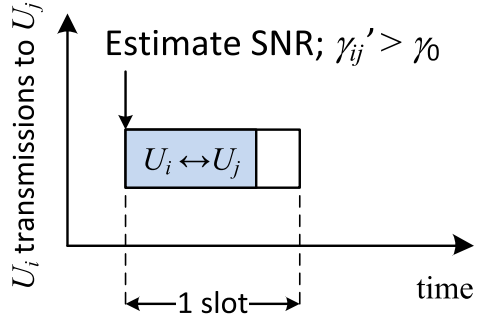

(b)

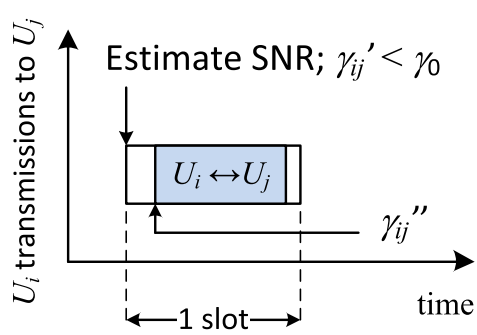

(c)

Fig. 2 a-c Scheduling in the internode communication

cardinality of the set with different combinations of $N_{b}$ elements, which are in the set $S_{i}$, and $b_{i j}$ the probability of $(i \leftrightarrow j)$ being blocked.

If all blockage probabilities are equal (i.e. $b_{i j}=b_{i}$ ), then (2) reduces to a binomial distribution

$$
\operatorname{Pr}\left(N_{b}\right)=\left(\begin{array}{c}
M-1 \\
N_{b}
\end{array}\right) b_{i}^{N_{b}}\left(1-b_{i}\right)^{M-N_{b}-1} .
$$

\section{Probability of successful internode decoding}

The probability that $U_{i}$ will successfully decode the packet from another node through an internode channel is the probability that the instantaneous internode SNR is greater than the given threshold, $\gamma_{0}$. An optimal protocol threshold $\gamma_{T H}$ which minimizes OP exists, and it is equal to the outage threshold $\gamma_{0}[13,17]$. In the case of correlated Rayleigh fading, the successful decoding probability takes the form

$$
p_{\bar{\gamma}_{i j}}=1-\int_{0}^{\gamma_{0}} \int_{0}^{\gamma_{0}} p_{\gamma_{i j}{ }^{\prime}, \gamma_{i j^{\prime \prime}}}\left(\gamma_{i j}{ }^{\prime}, \gamma_{i j}{ }^{\prime \prime}\right) d \gamma_{i j}{ }^{\prime} d \gamma_{i j}{ }^{\prime \prime},
$$

where $p_{\gamma_{i j}{ }^{\prime}, \gamma_{i j}{ }^{\prime \prime}}\left(\gamma_{i j}{ }^{\prime}, \gamma_{i j}{ }^{\prime \prime}\right)$ is the joint PDF of two correlated exponential random variables, given by (1).

Integration over the variable $\gamma_{i j}^{\prime}$, presented in [17] (eq. (9.279)) and [18] results in a closed-form expression that includes the Marcum- $Q$ function, defined in [19] (eq. (2-1122)). Integration over the second variable does not yield a closed-form expression.
To get a more desirable solution, the following derivation uses a series expansion of the zeroth order modified Bessel function of the first kind [20] (eq. (447.1))

$$
I_{0}(z)=\sum_{m=0}^{\infty} \frac{1}{(m !)^{2}}\left(\frac{z}{2}\right)^{2 m},
$$

and the joint PDF given in (1) can be rewritten as

$$
\begin{aligned}
& p_{\gamma_{i j}{ }^{\prime}, \gamma_{i j}{ }^{\prime \prime}}\left(\gamma_{i j}{ }^{\prime}, \gamma_{i j}{ }^{\prime \prime}\right)=\frac{1}{(1-\rho) \bar{\gamma}_{i j}^{2}} e^{-\frac{\gamma_{i j}{ }^{\prime}+\gamma_{i j}{ }^{\prime \prime}}{(1-\rho) \gamma_{i j}}} \\
& \times \sum_{m=0}^{\infty} \frac{1}{(m !)^{2}}\left(\frac{\rho \gamma_{i j}{ }^{\prime} \gamma_{i j}{ }^{\prime \prime}}{(1-\rho)^{2} \bar{\gamma}_{i j}^{2}}\right)^{m} .
\end{aligned}
$$

Substituting (1) with (6), (4) can be written in the form

$$
\begin{gathered}
p_{\bar{\gamma}_{i j}}=1-\sum_{m=0}^{\infty} \frac{\rho^{m}}{m !^{2}(1-\rho)^{2 m+1} \bar{\gamma}_{i j}^{2 m+2}} \\
\times \int_{0}^{\gamma_{0}}\left(\gamma_{i j}\right)^{m} e^{-\frac{\gamma_{i j} j^{\prime}}{(1-\rho) \hat{\gamma}_{i j}}} d \gamma_{i j}{ }^{\prime} \int_{0}^{\gamma_{0}}\left(\gamma_{i j}{ }^{\prime \prime}\right)^{m} e^{-\frac{\gamma_{i j}{ }^{\prime \prime}}{(1-\rho) \bar{\gamma}_{i j}}} d \gamma_{i j}{ }^{\prime \prime} .
\end{gathered}
$$

The integrals can be directly solved using [20] (eq. (3.351)); hence, the probability that a cooperating node will successfully decode a data packet from the node $U_{i}$ is derived in the form of

$$
\begin{aligned}
& p_{\overline{\gamma_{i j}}}=1-(1-\rho) \sum_{m=0}^{\infty} \rho^{m} \\
& \times\left(1-\frac{1}{m !} \Gamma\left(m+1, \frac{\gamma_{0}}{(1-\rho) \overline{\gamma_{i j}}}\right)\right)^{2},
\end{aligned}
$$


with $\Gamma(\cdot, \cdot)$ denoting the incomplete Gamma function, defined in [20] (eq. (8.350.2)). The infinite sum in (8) converges very rapidly, as shown in Table 1.

The expression in (8) gives exact numerical results using only a few terms in the infinite sum. For instance, to achieve accuracy at the 6th significant digit, only three terms are required for $\rho=0.2$, and 12 terms for $\rho=$ 0.9 when average internode SNR is $\bar{\gamma}_{i j}=0 \mathrm{~dB}$. As $\bar{\gamma}_{i j}$ increases, less terms give accurate results, and for $\bar{\gamma}_{i j}=15 \mathrm{~dB}$, just one term is sufficient for $\rho=0.2$ and two terms for $\rho=0.9$.

\section{Outage probability analysis}

In order to determine the OP in the system, we analyse the cases of full and partial cooperation, respectively. Full cooperation is the ideal case, and it assumes that node $U_{i}$ has successfully decoded all packets from the other cooperating nodes, i.e. error-free communication. The other case, denoted as partial cooperation, models the realworld system behaviour. In partial cooperation, node $U_{i}$ can fail to decode some of the packets from other nodes due to insufficient received SNR. Node $U_{i}$ will therefore re-send its own packet copies to the destination, replacing the packets from other nodes it has failed to decode. Furthermore, the overall OP will depend on the number of successfully decoded packets as well as the number of cooperating nodes (nodes which links are not blocked).

$n$ denotes the number of packets copies that the destination receives directly from node $U_{i}$ in the second stage of cooperation. Similar to (2), $n$ is determined by

$$
\operatorname{Pr}(N=n)=\operatorname{Pr}(n)=\sum_{\substack{S_{c} \subseteq S_{i} \\\left|S_{c}\right|=n}} \prod_{\lambda \in S_{i}}\left(1-p_{\bar{\gamma}_{i \lambda}}\right) \prod_{\kappa \in S_{i} \backslash S_{c}} p_{\bar{\gamma}_{i \kappa}},
$$

with $n=0,1, \ldots, M-1$. Similar to (2), $S_{c}$, the subset of $S_{i}$, denotes the number of packet copies that node $U_{i}$ re-sends in the second stage of cooperation, and $\left|S_{c}\right|$ is the cardinality of the set with different combinations of $n$ elements.

For the case when all $p_{\bar{\gamma}_{i j}}$ are equal, (9) has a binomial distribution and reduces to

$$
\operatorname{Pr}(n)=\left(\begin{array}{c}
M-1 \\
n
\end{array}\right)\left(1-p_{\bar{\gamma}_{i}}\right)^{n} p_{\bar{\gamma}_{i}}^{M-n-1} .
$$

The instantaneous SNR at the MRC receiver output at the destination is denoted as $\gamma_{\Sigma}$. To determine the OP of

Table 1 Convergence to the 6th significant digit of the sum in (8)

\begin{tabular}{llllll}
\hline$\gamma_{0}=-0.441 \mathrm{~dB}$ & $\rho=0.2$ & $\rho=0.4$ & $\rho=0.6$ & $\rho=0.8$ & $\rho=0.9$ \\
\hline $\bar{\gamma}_{i j}=0 \mathrm{~dB}$ & 3 & 4 & 6 & 10 & 12 \\
$\bar{\gamma}_{i j}=5 \mathrm{~dB}$ & 2 & 2 & 4 & 5 & 7 \\
$\bar{\gamma}_{i j}=10 \mathrm{~dB}$ & 1 & 1 & 1 & 2 & 4 \\
$\bar{\gamma}_{i j}=15 \mathrm{~dB}$ & 1 & 1 & 1 & 1 & 2 \\
\hline
\end{tabular}

the network in both cases, it is required to find the OPs $P_{\mathcal{F}}\left(\gamma_{\Sigma}\right)$ and $P_{\mathcal{P}}\left(\gamma_{\Sigma}\right)$, where $\mathcal{F}$ and $\mathcal{P}$ denote full and partial cooperation, respectively. The full and partial outages are conditioned on the number of nodes, which in the case of link blockage has a probability given by (2).

In the case of full cooperation, node $U_{i}$ has decoded all the packets from other nodes. In this case, the destination combines $M$ copies of the packet, and also, it holds that $n=0$. Similar to [17] (eq. (9.5)), the conditional OP is

$$
P_{\mathcal{F}}\left(\gamma_{0} \mid M\right)=1-\frac{1}{(M-1) !} \Gamma\left(M, \frac{\gamma_{0}}{\bar{\gamma}_{u}}\right) .
$$

In the case of partial cooperation, the number of packets which node $U_{i}$ transmits in the second stage is $n>0$. In this case, just from node $U_{i}$, the destination receives $n+1$ copies of the packet, where each packet has an average SNR of $\bar{\gamma}_{u}$. The destination then applies MRC on the $n+1$ copies (which can be written as a single copy at an SNR value of $\left.(n+1) \bar{\gamma}_{u}\right)$ with the remaining $M-n-1$ copies from other cooperating nodes. The OP, conditioned on $M$ and $n$, can be derived from the moment generating function (MGF) of $\gamma_{\Sigma}$. Using partial fractions [20] and taking the inverse Laplace transform, the expression for the conditional OP for partial cooperation, similar to [11] (eq. (6)), can be written as

$$
\begin{aligned}
& P_{\mathcal{P}}\left(\gamma_{0} \mid M, n\right)=1-\exp \left(-\frac{\gamma_{0}}{(n+1) \bar{\gamma}_{u}}\right)\left(\frac{n+1}{n}\right)^{M-n-1} \\
& +\exp \left(-\frac{\gamma_{0}}{\bar{\gamma}_{u}}\right)^{M-n-2} \sum_{k=0}^{M-2} \frac{1}{k !}\left(\frac{\gamma_{0}}{\bar{\gamma}_{u}}\right)^{k}\left[\left(\frac{n+1}{n}\right)^{M-n-k-1}-1\right]
\end{aligned}
$$

A piecewise general expression for the $\mathrm{OP}$ can be expressed as ([11], eq. (7))

$$
P_{O}\left(\gamma_{0} \mid M, n\right)= \begin{cases}P_{\mathcal{F}}\left(\gamma_{0} \mid M\right), & n=0 \\ P_{\mathcal{P}}\left(\gamma_{0} \mid M, n\right), & n>0\end{cases}
$$

The overall OP for a network of $M$ nodes without link blockage can be obtained by averaging (13) over the number of packets received by the destination directly from node $U_{i}$ in the second stage of cooperation as

$$
P_{O}\left(\gamma_{0}\right)=\sum_{n=0}^{M-1} \operatorname{Pr}(n) P_{O}\left(\gamma_{0} \mid M, n\right) .
$$

Furthermore, taking into account the number of links being blocked, $N_{b}$, the OP for the blocked case is obtained by averaging (14) over the distribution of number of blocked links as

$$
P_{O}\left(\gamma_{0}\right)=\sum_{N_{b}=0}^{M-1} \sum_{n=0}^{M-1} \operatorname{Pr}\left(N_{b}\right) \operatorname{Pr}(n) P_{O}\left(\gamma_{0} \mid M-N_{b}, n\right) .
$$

\section{Asymptotic analysis}

We analyse the asymptotic behaviour of the network at high average SNR to gain a better insight on outage performance. As both average uplink and average internode 
SNR increase, we examine the relation of $\bar{\gamma}_{i}$ and $\bar{\gamma}_{u}$ and present OP approximations for two distinct cases, the former being high average internode $\left(\bar{\gamma}_{i} \gg \bar{\gamma}_{u}\right)$ and the latter being the high average uplink $\left(\bar{\gamma}_{u} \gg \bar{\gamma}_{i j}\right)$ regime. In both cases, all the average internode SNRs are assumed to be equal, i.e. $\bar{\gamma}_{i j}=\bar{\gamma}_{i}$. In addition, as the average uplink SNR goes to infinity, we observe the diversity and coding gains, as the OP can be written as $\tilde{P}_{O}\left(\gamma_{0}\right)=\left(\mathcal{G}_{c} \bar{\gamma}_{u}\right)^{-\mathcal{G}_{d}}$, with $\mathcal{G}_{c}$ denoting the coding gain and $\mathcal{G}_{d}$ the diversity gain. We first solve OP, coding and diversity gains for the non-blocked case, and then extend to the blocked case by averaging over the number of blocked links.

The approximate asymptotic expressions for the full and partial conditional outage probabilities when $\bar{\gamma}_{u} \gg \gamma_{0}$, which hold for both cases, are given, respectively, as ([11], eq. (15))

$$
\tilde{P}_{\mathcal{F}}\left(\gamma_{0} \mid M\right)=\frac{1}{M !}\left(\frac{\gamma_{0}}{\bar{\gamma}_{u}}\right)^{M}
$$

and ([11], eq. (16))

$$
\tilde{P}_{\mathcal{P}}\left(\gamma_{0} \mid M, n\right)=\frac{1}{(M-n-1) !(n+1)}\left(\frac{\gamma_{0}}{\bar{\gamma}_{u}}\right)^{M-n} \text {. }
$$

We utilize the expressions above in the derivation of the asymptotic results.

\subsection{Case I: $\bar{\gamma}_{i} \gg \bar{\gamma}_{u}$}

In the first case, we assume that the average internode SNR is greater than the average uplink SNR (i.e., $\bar{\gamma}_{i} \gg \bar{\gamma}_{u}$ ), and internode communication is fully reliable. There are no delayed transmissions in the internode protocol. This in turn also results that in the second phase of cooperation, node $U_{i}$ does not re-send its own packets.

The dominating term in the expression for successful decoding in the internode channel in (8) is the first term in the sum, and we can write

$$
\tilde{p}_{\bar{\gamma}_{i}}=1-(1-\rho)\left(1-\Gamma\left(1, \frac{\gamma_{0}}{(1-\rho) \bar{\gamma}_{i}}\right)\right)^{2} .
$$

Furthermore, from $\bar{\gamma}_{i} \gg \bar{\gamma}_{u} \gg \gamma_{0}$, we can apply the approximation $\frac{1}{N !} \Gamma(N+1, z) \approx 1-\frac{z^{N+1}}{\Gamma(N+2)}$, and after some mathematical manipulations, we obtain the approximation for successful internode decoding as

$$
\tilde{p}_{\bar{\gamma}_{i}}=1-\frac{1}{(1-\rho)}\left(\frac{\gamma_{0}}{\bar{\gamma}_{i}}\right)^{2}
$$

By inserting (16) and (19) into (14), the expression for asymptotic OP without link blockage can therefore be written as

$$
\tilde{P}_{O}\left(\gamma_{0}\right)=\frac{1}{M !}\left(\frac{\gamma_{0}}{\bar{\gamma}_{u}}\right)^{M}\left(1-\frac{1}{(1-\rho)}\left(\frac{\gamma_{0}}{\bar{\gamma}_{i}}\right)^{2}\right)^{M-1} .
$$

As $\bar{\gamma}_{u}$ goes to infinity, we observe the coding and diversity gains. Directly from (20), and using $\bar{\gamma}_{i} \gg \gamma_{0}$, we can obtain the gains as

$$
\mathcal{G}_{c_{i}}=\frac{(M !)^{1 / M}}{\gamma_{0}}, \quad \mathcal{G}_{d_{i}}=M
$$

Next, we will be examining the impact of link blockage. Averaging the OP (20) over the number of blocked links, we obtain the OP in the high average internode regime in the case of link blockage as

$$
\begin{aligned}
\tilde{P}_{O}\left(\gamma_{0}\right) & =\sum_{N_{b}=0}^{M-1} \operatorname{Pr}\left(N_{b}\right) \frac{1}{\left(M-N_{b}\right) !}\left(\frac{\gamma_{0}}{\bar{\gamma}_{u}}\right)^{M-N_{b}} \\
& \times\left(1-\frac{1}{(1-\rho)}\left(\frac{\gamma_{0}}{\bar{\gamma}_{i}}\right)^{2}\right)^{M-N_{b}-1} .
\end{aligned}
$$

Bearing in mind that there exists a finite probability that all internode links are blocked, outage performance will be dominated by the case when the only active link is the direct link from node $U_{i}$ to the destination. Diversity gain is therefore reduced to one, and the coding gain is obtained from (22) when $N_{b}=M-1$, i.e. when all internode links are blocked. The gains are therefore

$$
\mathcal{G}_{c_{i}}=\frac{1}{\gamma_{0} b_{i}^{M-1}}, \quad \mathcal{G}_{d_{i}}=1
$$

\subsection{Case II: $\bar{\gamma}_{u} \gg \bar{\gamma}_{i}$}

In the second case, the average uplink SNR is greater than the average internode SNR $\left(\bar{\gamma}_{u} \gg \bar{\gamma}_{i}\right)$. Performance is determined by the case when all internode channels fail, and nodes cannot successfully decode packets originating from node $U_{i}$. In this case, all packets are re-sent directly from the initial node $U_{i}$ in the second stage of cooperation. This behaviour can be analytically expressed as, when calculating the OP, the only term in the sum w.r.t. $n$ in (14) is the last, $(M-1)$ st term. The outage probability can therefore be calculated inserting (17) into (14) for $n=M-1$, without blockage, as

$$
\tilde{P}_{O}\left(\gamma_{0}\right)=\frac{1}{M}\left(\frac{\gamma_{0}}{\bar{\gamma}_{u}}\right)\left(1-p_{\bar{\gamma}_{i}}\right)^{M-1} .
$$

It is worth noting that, in the expression above, $p_{\overline{\gamma_{i j}}}$ cannot be approximated to the first term as in (19), as the ratio between $\bar{\gamma}_{i}$ and $\gamma_{0}$ can be arbitrary. The approximation for successful internode packet decoding from (19) is accurate only for $\bar{\gamma}_{i} \gg \gamma_{0}$. Rather, the exact probability of successful packet decoding in (8) is used.

In a similar fashion to the internode regime, from (24), the coding and diversity gains for high average uplink SNR regime, when $\bar{\gamma}_{u} \rightarrow \infty$, are given respectively, as

$$
\mathcal{G}_{c_{u}}=\frac{M}{\gamma_{0}\left(1-p_{\overline{\gamma_{i}}}\right)^{M-1}}, \quad \mathcal{G}_{d_{u}}=1 .
$$

However, for the ratio $\bar{\gamma}_{i} \gg \gamma_{0}$, in accordance with Table 1, only the first term in (8) is sufficient, and we can 
apply the approximation for successful internode channel decoding (18). For this case, $\mathcal{G}_{d_{u}}$ remains equal to one, but one can see more clearly the impact of the correlation coefficient $\rho$ on $\mathcal{G}_{c_{u}}$ as

$$
\mathcal{G}_{c_{u}}=\frac{M}{\gamma_{0}\left(\frac{1}{(1-\rho)}\left(\frac{\gamma_{0}}{\bar{\gamma}_{i}}\right)^{2}\right)^{M-1}}, \quad \mathcal{G}_{d_{u}}=1 .
$$

Finally, when we take into account the probability of link blockage, we can write the asymptotic expression for the high average uplink SNR regime, from (24), as

$$
\begin{aligned}
\tilde{P}_{O}\left(\gamma_{0}\right) & =\sum_{N_{b}=0}^{M-1} \operatorname{Pr}\left(N_{b}\right) \frac{1}{\left(M-N_{b}\right)} \\
& \times\left(\frac{\gamma_{0}}{\bar{\gamma}_{u}}\right)\left(1-p_{\bar{\gamma}_{i}}\right)^{M-N_{b}-1} .
\end{aligned}
$$

From (27), we can directly obtain the coding and diversity gains for the high average uplink SNR regime with link blockage as

$$
\mathcal{G}_{c_{u}}=\frac{1}{\gamma_{0}} \frac{1}{\sum_{N_{b}=0}^{M-1} \operatorname{Pr}\left(N_{b}\right) \frac{\left(1-p_{\overline{\gamma_{i}}}\right)}{\left(M-N_{b}\right)}}{ }^{M-N_{b}-1} \mathcal{G}_{d_{u}}=1 .
$$

\section{Numerical results}

The expressions derived in the previous section are used to efficiently compute numerical values of OP for any practical value of average SNR over internode and uplink channel, correlation coefficient and network dimension. The threshold for successfully decoding packets depends on the coding scheme used and on the packet length $L$, as derived in [21]. We set the optimum threshold to $\gamma_{0}=-0.441 \mathrm{~dB}$, which describes a network where each node encodes a packet of length $L=512 \mathrm{~b}$ using a convolutional coding scheme with one-half code rate with octal generator polynomials $(1,17 / 15)$, [21]. In addition, average internode SNRs are assumed to be equal due to simpler mathematical tractability, except in Fig. 5, where average internode SNRs follow an exponential power profile [22].

Figure 3 shows the OP as a function of average internode SNR, for various values of average uplink SNR and correlation coefficient $\rho$. Also, as a comparison, results from [11] and [13] are given. The results for the full correlation limiting case coincide with the results when the thresholdbased protocol is not used, as presented in [11]. These additional curves from [11] and [13] act as upper and lower bounds, for the limiting cases when $\rho$ approaches one (full correlation) and zero (no correlation), respectively. For higher average internode SNR, on the average, internode channel state will be above the threshold. This results in less packet errors between the nodes, obtaining better performance until a floor is reached. The impact of correlation is dominant in the low average internode SNR

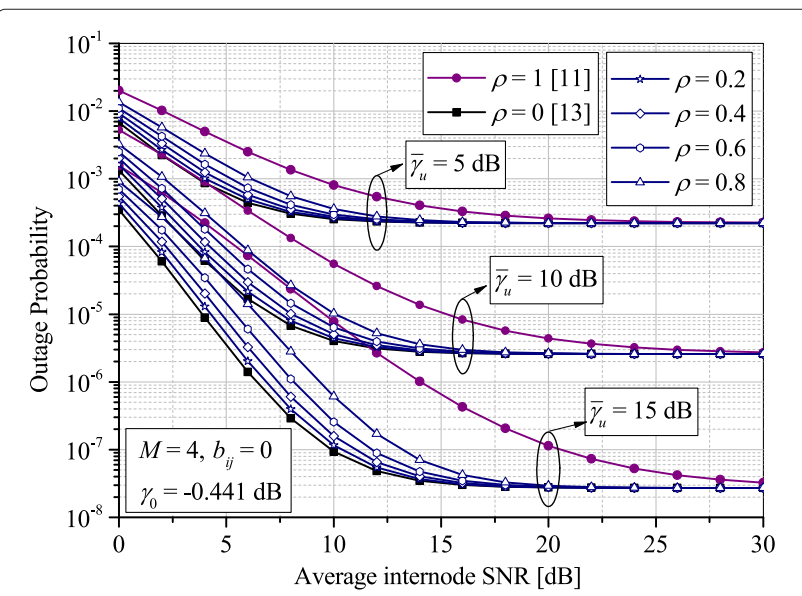

Fig. 3 Outage probability vs. average internode SNR for different $\rho$

regime. For instance, for $\bar{\gamma}_{12}=10 \mathrm{~dB}$, when $\rho$ increases from 0 to $1, P_{0}$ increases 85.1 times for $\bar{\gamma}_{u}=15 \mathrm{~dB}$ and only 3.5 times for $\bar{\gamma}_{u}=5 \mathrm{~dB}$. For greater values of $\bar{\gamma}_{12}$, correlation does not have an impact on outage probability. All curves corresponding to different values of $\rho$ tend to an outage floor that does not depend on $\rho$.

Figure 4 further shows outage probability dependence on the correlation coefficient for different values of average internode SNR for a network without and with link blockage. As expected, by increasing the correlation between fading samples in internode communication, outage performance is degraded. However, as shown in the figure, by increasing $\bar{\gamma}_{i}$ the probability of delayed internode packet transmission decreases, the impact of correlation on the system performance becomes less significant. The difference in OP when link blockage exists, when compared to the non-blocked case, is more apparent for greater values of $\bar{\gamma}_{i}$.

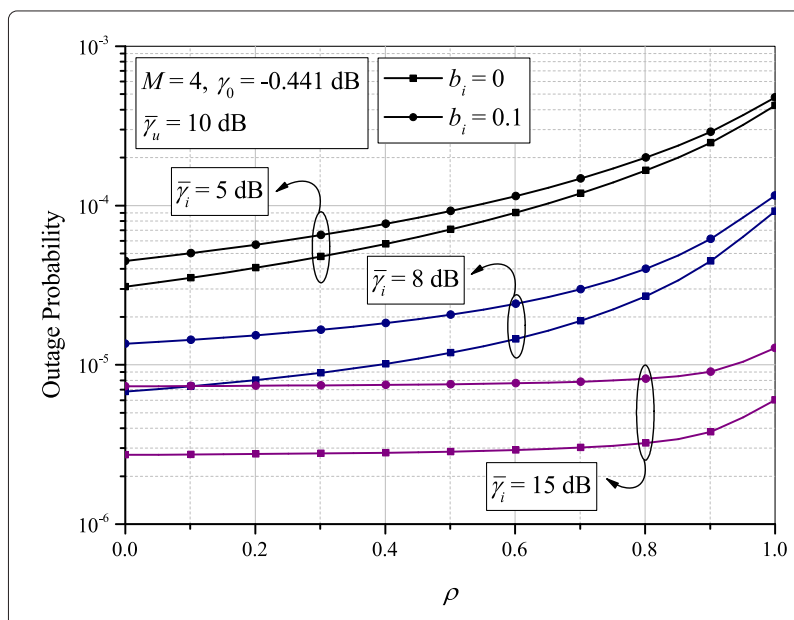

Fig. 4 Outage probability dependence on correlation coefficient for different values of average internode SNR $\bar{\gamma}_{i}$ 
In Fig. 5, average internode SNRs are unequal and follow the exponential power profile [22], given as $\bar{\gamma}_{1 j}=\bar{\gamma}_{1 i} e^{-\delta(j-2)}$, where $i, j=1, \ldots, M, i \neq j$ and $\delta$ is an non-negative integer. Increasing $\delta$, outage saturation is reached at higher $\bar{\gamma}_{12}$. For $\delta=0$, all internode SNRs are equal and the outage floor is reached at $\bar{\gamma}_{12}=20 \mathrm{~dB}$. Outage probability increases with increasing $\delta$, and the required $\bar{\gamma}_{12}$ to reach the floor shifts from 20 to $28 \mathrm{~dB}$ as $\delta$ goes from 0 to 1 .

Figure 6 shows outage probability dependence on average uplink SNR for different number of nodes $M$ and correlation coefficient $\rho$. Average internode SNRs increase simultaneously with the average uplink SNRs. The impact of correlation is stronger at lower average SNR and is more pronounced for higher network dimension. For instance, at $\bar{\gamma}_{u}=5 \mathrm{~dB}$, as $\rho$ decreases from 0.9 to 0.1 outage probability drops only 1.266 times for $M=2$, while for $M=5$, outage is 4.83 times lower. When the blockage probability is different from zero, in the high average SNR regime, correlation has no impact on outage for any network size. Additionally, the finite blockage probability causes the slope of the outage curves to approach diversity order one at high SNR for any number of nodes (because the node's uplink channel is always available) and diverge from the non-blocked case, presented by the dashed curves. Note that in the non-blocked case, outage curves have diversity order $M$.

The effects of different blockage probabilities are shown in Fig. 7. Even for high average uplink SNR $\bar{\gamma}_{u}$, the impact of link blockage is dominant. As $b_{i j}$ increases, the floor is reached at a smaller value of OP. For instance, at $b_{i j}=$ 0.01 , the outage floor of $2.6 \times 10^{-8}$ is reached at about $\bar{\gamma}_{u}=20 \mathrm{~dB}$, while at $b_{i j}=0.1$, the floor of $10^{-5}$ is reached at $\bar{\gamma}_{u}=15 \mathrm{~dB}$. In the asymptotic average internode SNR regime with fixed average uplink SNR, one can notice that

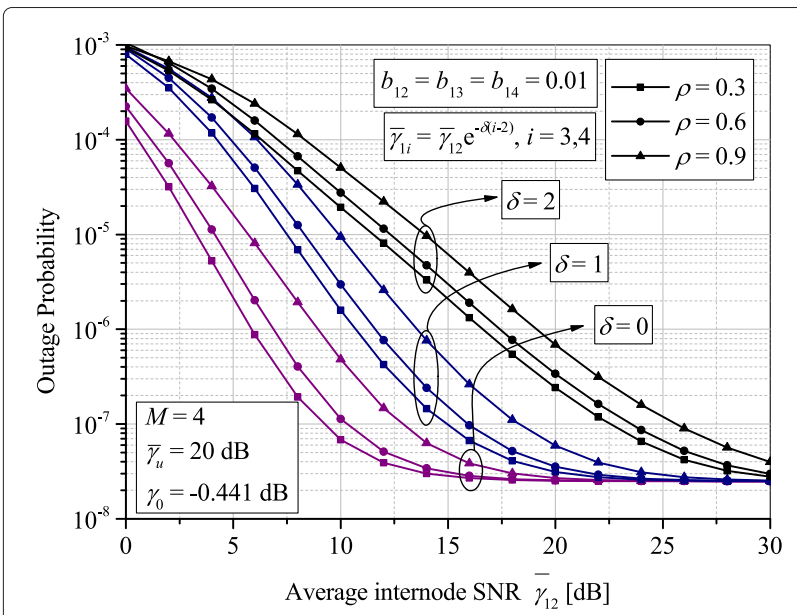

Fig. 5 Outage probability vs. average internode $\bar{\gamma}_{12}$ SNR for different $\delta$

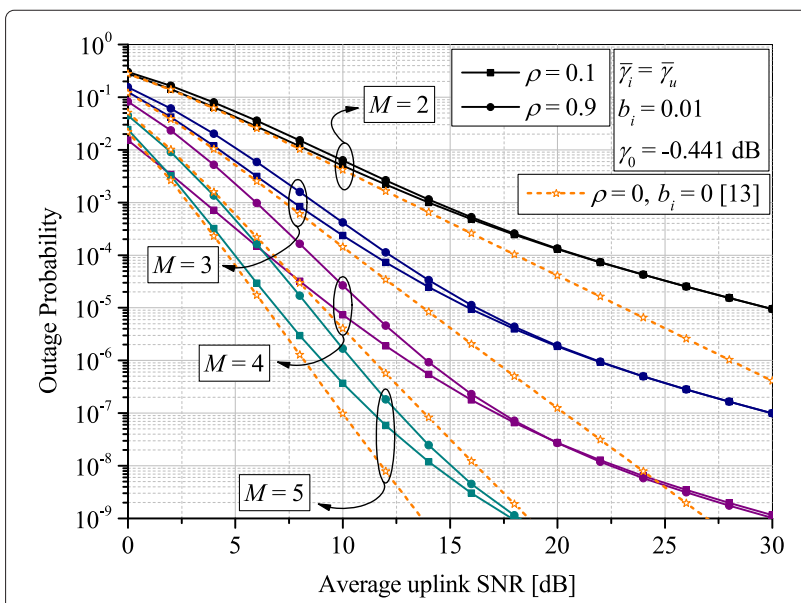

Fig. 6 Outage probability vs. average uplink SNR for different number of nodes and different $\rho$

the outage does not depend on internode communication. The value of the outage floor can be obtained using (16), as

$$
P_{\mathrm{FLOOR}}=\sum_{N_{b}=0}^{M-1} \operatorname{Pr}\left(N_{b}\right) \tilde{P}_{\mathcal{F}}\left(\gamma_{0} \mid M-N_{b}\right) .
$$

When plotting the OP dependence on average uplink SNR in Fig. 8, the effect of correlation in the internode communication protocol can be observed. We also plot the asymptotic expressions for both high average internode and average uplink SNRs. Orange curves represent the high average internode regime, are plotted for $\bar{\gamma}_{i}=15$ $\mathrm{dB}$ and are a good match for the exact curves until $\bar{\gamma}_{u}=12$ $\mathrm{dB}$, as expected. On the other hand, for $\bar{\gamma}_{i}=5 \mathrm{~dB}$, the teal curves, for the high average uplink regime are a good match beyond $\bar{\gamma}_{u}=16 \mathrm{~dB}$ and for $\bar{\gamma}_{i}=15 \mathrm{~dB}$ beyond $\bar{\gamma}_{u}=30 \mathrm{~dB}$.

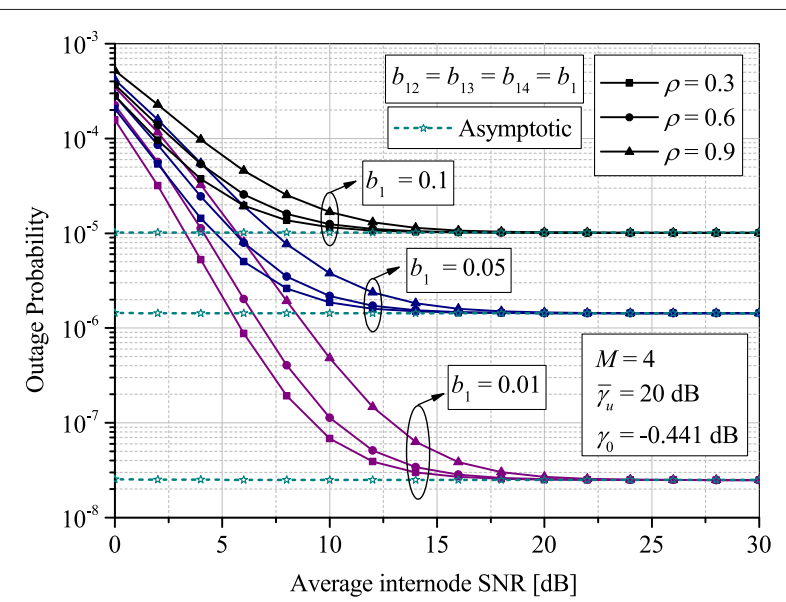

Fig. 7 Outage probability vs. average uplink SNR for different $\bar{\gamma}_{i}$ 


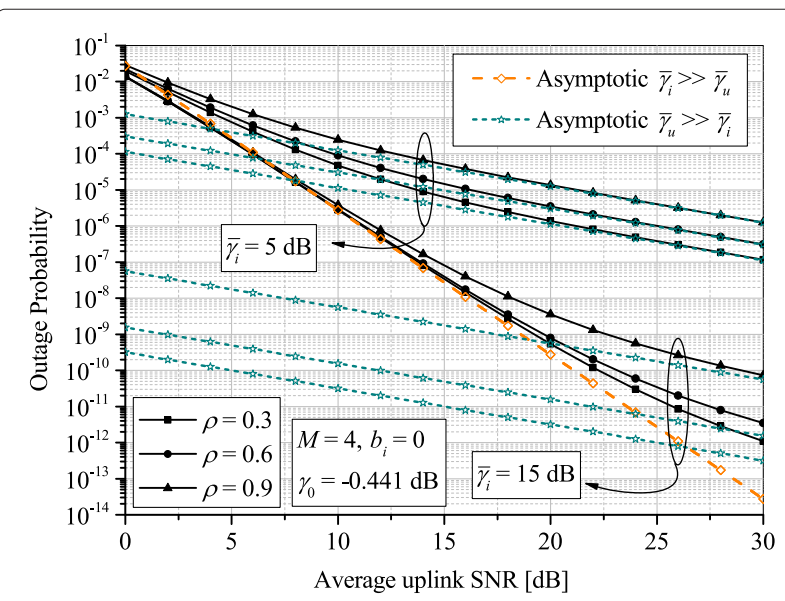

Fig. 8 Outage probability vs. average internode SNR for different $b_{i}$

One can also notice the diversity and coding gains on the asymptotic curves. As expected, at high average internode SNR, the diversity gain is determined by the slope of the orange curve and is equal to $M$, while for high average uplink SNR, all teal curves are diversity order one. Coding gain in the high average internode regime does not depend on the correlation in the internode protocol (all three orange curves are overlapping), while $\rho$ has a significant impact in the high average uplink SNR regime.

Finally, Fig. 9 shows outage dependence on average uplink SNR for different values of node blockage $b_{i}$. Exact and asymptotic results are presented. As $b_{i}$ increases, OP converges to a single-link case (with no cooperation). The additional, single-link curve is plotted as well for comparison. Furthermore, asymptotic results, in both the high average internode SNR from (22) and high average uplink SNR from (27), converge to the exact value of the OP as $b_{i}$ increases. The slope of the $\bar{\gamma}_{i} \gg \bar{\gamma}_{u}$ asymptotic curves changes from being very high at low $\bar{\gamma}_{u}$ but reduces to first

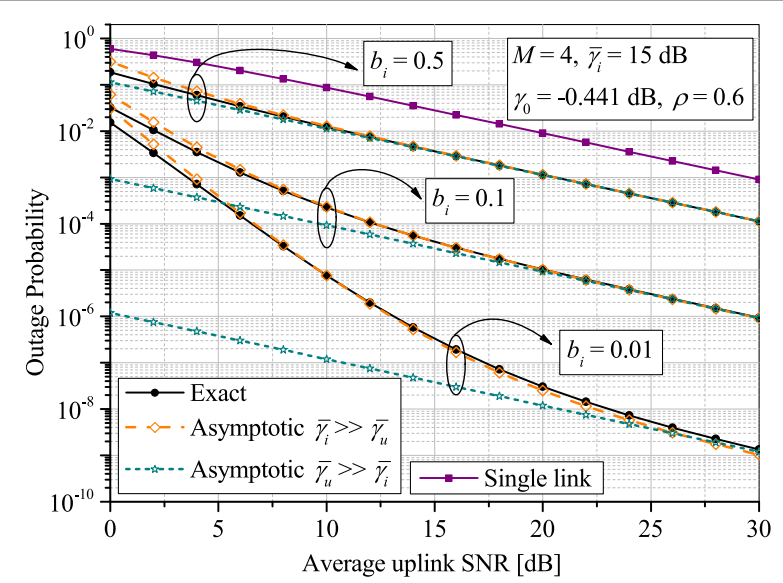

Fig. 9 Outage probability vs. average uplink SNR for different $b_{i}$ order diversity as $\bar{\gamma}_{u}$ increases. For instance, for $b_{i}=0.01$, the slope tends to full-order diversity (as in Fig. 8, with no blockage) for $\bar{\gamma}_{u}<15 \mathrm{~dB}$. However, due to link blockage, this slope tends to first-order diversity as $\bar{\gamma}_{u}$ goes above 15 $\mathrm{dB}$. As $b_{i}$ decreases, the slope turning point tends to the fixed value of the average internode SNR.

\section{Conclusions}

In this paper, we have investigated DF cooperative networks prone to link blockage over temporally correlated Rayleigh fading between nodes and derived novel exact and asymptotic expressions for outage probability. Based on these expressions, we have analysed the outage probability dependence on different channel and network parameters, as are link blockage probability, number of nodes in the network, average SNR over uplink and internode channels, as well as temporary fading correlation. Asymptotic results demonstrate the interplay of high average uplink and internode SNRs on outage performance. The derived asymptotic expressions, which are more tractable from an engineering point of view, emphasize network behaviour at high average SNR regimes.

The presented numerical results demonstrate that fading correlation over the internode channels has a stronger influence on outage for greater values of average uplink SNR, but at low ranges of average internode SNR. At high average internode SNR, the impact of correlation even diminishes. Moreover, as the network size increases, correlation has greater impact on outage probability.

The results have shown that the presence of finite link blockage causes significant performance degradation, reducing the diversity order of the system to a single-link case with diversity order one. This non-negligible performance loss is present regardless of the correlation coefficient in the threshold-based internode protocol. Furthermore, by increasing link blockage probability by one order of magnitude, the outage probability is increased by several orders of magnitude.

\section{Competing interests}

The authors declare that they have no competing interests.

\section{Acknowledgements}

This paper was supported in part by the Norwegian Ministry for Foreign Affairs under the project "Norwegian, Bosnian and Serbian cooperation platform for university and industry ICT R \& D" and in part by the Ministry of Science of Republic of Serbia under technology development project TR-32028 "Advanced techniques for efficient use of spectrum in wireless systems". The authors would like to thank the reviewers and the associate editor for the valuable comments that improved the quality of this paper.

Received: 21 April 2015 Accepted: 29 March 2016

Published online: 05 April 2016

\section{References}

1. J Laneman, D Tse, GW Wornell, Cooperative diversity in wireless networks: efficient protocols and outage behavior. IEEE Trans. Inf. Theory. 50, 3062-3080 (2004) 
2. MR Souryal, B Vojcic, in Proceedings of the IEEE International Conference on Acoustics, Speech and Signal Processing, ICASSP 2006. Performance of amplify-and-forward and decode-and-forward relaying in Rayleigh fading with turbo codes (IEEE, Toulouse, France, 2006). http://ieeexplore.ieee. org/xpl/articleDetails.jsp?arnumber $=1661060$

3. AK Sadek, W Su, KR Liu, in Proceedings of the IEEE Global Telecommunications Conference, GLOBECOM'05. Clustered cooperative communications in wireless networks, vol. 3 (IEEE, Missouri, USA, 2005), p. 5. http://ieeexplore.ieee.org/xpl/articleDetails.jsp?arnumber=1577835

4. Y Zhao, R Adve, TJ Lim, Outage probability at arbitrary SNR with cooperative diversity. IEEE Commun. Lett. 9(8), 700-702 (2005)

5. K Woradit, TQ Quek, W Suwansantisuk, H Wymeersch, L Wuttisittikulkij, MZ Win, Outage behavior of selective relaying schemes. IEEE Trans. Wirele. Commun. 8(8), 3890-3895 (2009)

6. S Ren, KB Letaief, JRB de Marca, Outage reduction in cooperative networks with limited feedback. IEEE Trans. Commun. 58(3), 748-752 (2010)

7. D Nguyen, Q Nguyen, TC Chung, in Proceedings of International Conference on Advanced Technologies for Communications (ATC). Outage probability analysis of cooperative diversity DF relaying under Rayleigh fading (IEEE, Da Nang, Vietnam, 2011), pp. 116-120. http://ieeexplore. ieee.org/xpl/articleDetails.jsp?arnumber $=6027448$

8. OJ Pandey, A Trivedi, MK Shukla, in Tenth International Conference on Wireless and Optical Communications Networks (WOCN). Outage performance of decode-forward and amplify-forward protocols in cooperative wireless communication (IEEE, Bhopal, India, 2013), pp. 1-5. http://ieeexplore.ieee.org/xpl/articleDetails.jsp?arnumber=6616196

9. H Nguyen, N Tran, H Nguyen, Performance analysis of adaptive decode-and-forward relaying in noncoherent cooperative networks. EURASIP J. Wirel. Commun. Netw. 2013(1), 281 (2013)

10. L-Q-V Tran, O Berder, O Sentieys, On the performance of distributed space-time coded cooperative relay networks based on inter-relay communications. EURASIP J. Wirel. Commun. Netw. 2013(1), 239 (2013)

11. I Chatzigeorgiou, W Guo, IJ Wassell, RA Carrasco, Exact and asymptotic outage probability analysis for decode-and-forward networks. IEEE Trans. Commun. 59(2), 376-381 (2011)

12. AM Cvetkovic, K Kansanen, GT Djordjevic, in Proceedings of 20th Telecommunications Forum (TELFOR). Outage analysis for uncoordinated decode-and-forward networks over Nakagami-m channels (IEEE, Belgrade, Serbia, 2012), pp. 490-493. http://ieeexplore.ieee.org/xpl/ articleDetails.jsp?arnumber $=6419254$

13. NM Zdravkovic, AM Cvetkovic, GT Djordjevic, K Kansanen, in Proceedings of 21st Telecommunications Forum (TELFOR). Outage probability of decode-and-forward network with threshold-based protocol over Rayleigh fading, (2013), pp. 315-318

14. E Tanghe, W Joseph, L Verloock, L Martens, H Capoen, K Van Herwegen, W Vantomme, The industrial indoor channel: large-scale and temporal fading at 900, 2400, and 5200 Mhz. IEEE Trans. Wirel. Commun. 7(7), 2740-2751 (2008)

15. Q Braet, D Plets, W Joseph, L Martens, in Proceedings of 8th European Conference onAntennas and Propagation (EuCAP). Real-time path loss modelling for a more robust wireless performance, (2014), pp. 2109-2113

16. S Ivanov, E Nett, Localization-based radio model calibration for fault-tolerant wireless mesh networks. IEEE Trans. Ind. Informat. 9(1), 246-253 (2013)

17. MK Simon, M-S Alouini, Digital Communication over Fading Channels, vol. 95. (Wiley, New York, 2005)

18. AA Abu-Dayya, NC Beaulieu, Analysis of switched diversity systems on generalized-fading channels. IEEE Trans. Commun. 42(11), 2959-2966 (1994)

19. JG Proakis, Digital Communications, 4th edn. (McGraw-Hill Higher Education, New York, 2001)

20. I Gradshteyn, I Ryzhik, Table of Integrals, Series and Products, 5th edn. (Academic Press, New York, 1994)

21. I Chatzigeorgiou, IJ Wassell, R Carrasco, in Proceeding of the 42nd Annual Conference on Information Sciences and Systems, CISS. On the frame error rate of transmission schemes on quasi-static fading channels (IEEE, New Jersey, USA, 2008), pp. 577-581. http://ieeexplore.ieee.org/xpl/ articleDetails.jsp?arnumber $=4558591$

22. TS Rappaport, Wireless Communications: Principles and Practice, vol. 2. (Prentice Hall PTR, New Jersey, 1996)

\section{Submit your manuscript to a SpringerOpen ${ }^{\circ}$ journal and benefit from:}

- Convenient online submission

- Rigorous peer review

- Immediate publication on acceptance

- Open access: articles freely available online

- High visibility within the field

- Retaining the copyright to your article

Submit your next manuscript at $\gg$ springeropen.com 